\title{
Efficacy of Quadrivalent HPV Vaccine against HPV Infection and Disease in Males
}

\author{
Anna R. Giuliano, Ph.D., Joel M. Palefsky, M.D., Stephen Goldstone, M.D., Edson D. \\ Moreira Jr., M.D., Mary E. Penny, M.D., Carlos Aranda, M.D., Eftyhia Vardas, M.D., Harald \\ Moi, M.D., Heiko Jessen, M.D., Richard Hillman, M.D., Yen-Hwa Chang, M.D., Daron Ferris, \\ M.D., Danielle Rouleau, M.D., Janine Bryan, Ph.D., J. Brooke Marshall, Ph.D., Scott \\ Vuocolo, Ph.D., Eliav Barr, M.D., David Radley, M.S., Richard M. Haupt, M.D., and Dalya \\ Guris, M.D. \\ Risk Assessment, Detection, and Intervention Program, H. Lee Moffitt Cancer Center and \\ Research Institute, Tampa, FL (A.R.G.); Department of Medicine, University of California San \\ Francisco, San Francisco (J.M.P.); Mount Sinai School of Medicine, New York (S.G.); Associação \\ Obras Sociais Irmã Dulce and Oswaldo Cruz Foundation, Brazilian Ministry of Health, Bahia, \\ Brazil (E.D.M.); Instituto de Investigación Nutricional, Lima, Peru (M.E.P.); University Medical \\ Center, National Institute of Public Health, Morelos, Mexico (C.A.); Ndlela Research and Clinical \\ Trials Unit, Faculty of Health Sciences, University of the Witwatersrand, Johannesburg, South \\ Africa (E.V.); Olafia Sexually Transmitted Infections Clinic, Oslo University Hospital and Faculty of \\ Medicine, University of Oslo, Oslo (H.M.); Private Clinic for Infectious Diseases, Berlin (H.J.); \\ Sexually Transmitted Infections Research Centre, University of Sydney, Sydney (R.H.); Division \\ of Urology, Department of Surgery, Taipei Veterans General Hospital, Taipei, Taiwan (Y.-H.C.); \\ Medical College of Georgia, Augusta (D.F.); Centre de Recherche du Centre Hospitalier de \\ I'Université de Montréal, Montreal (D. Rouleau, D. Radley); and Merck, North Wales, PA (J.B., \\ J.B.M., S.V., E.B., R.M.H., D.G.)
}

\section{Abstract}

BACKGROUND—Infection with human papillomavirus (HPV) and diseases caused by HPV are common in boys and men. We report on the safety of a quadrivalent vaccine (active against HPV types $6,11,16$, and 18) and on its efficacy in preventing the development of external genital lesions and anogenital HPV infection in boys and men.

METHODS-We enrolled 4065 healthy boys and men 16 to 26 years of age, from 18 countries in a randomized, placebo-controlled, double-blind trial. The primary efficacy objective was to show that the quadrivalent HPV vaccine reduced the incidence of external genital lesions related to HPV-6, 11, 16, or 18. Efficacy analyses were conducted in a per-protocol population, in which subjects received all three vaccinations and were negative for relevant HPV types at enrollment, and in an intention-to-treat population, in which subjects received vaccine or placebo, regardless of baseline HPV status.

RESULTS-In the intention-to-treat population, 36 external genital lesions were seen in the vaccine group as compared with 89 in the placebo group, for an observed efficacy of $60.2 \%$ (95\% confidence interval [CI], 40.8 to 73.8 ); the efficacy was $65.5 \%$ (95\% CI, 45.8 to 78.6 ) for lesions

\footnotetext{
Copyright $\odot 2011$ Massachusetts Medical Society.

Address reprint requests to Dr. Giuliano at the Risk Assessment, Detection, and Intervention Program, H. Lee Moffitt Cancer Center and Research Institute, 12902 Magnolia Dr., MRC-CANCONT, Tampa, FL 33612, or at anna.giuliano@moffitt.org. Drs. Giuliano and Palefsky contributed equally to this work.

No other potential conflict of interest relevant to this article was reported.

Disclosure forms provided by the authors are available with the full text of this article at NEJM.org.
} 
related to HPV-6, 11, 16, or 18. In the per-protocol population, efficacy against lesions related to HPV-6, 11, 16, or 18 was 90.4\% (95\% CI, 69.2 to 98.1). Efficacy with respect to persistent infection with HPV-6, 11, 16, or 18 and detection of related DNA at any time was $47.8 \%$ (95\% CI, 36.0 to 57.6 ) and $27.1 \%$ (95\% CI, 16.6 to 36.3 ), respectively, in the intention-to-treat population and $85.6 \%$ (97.5\% CI, 73.4 to 92.9 ) and $44.7 \%$ (95\% CI, 31.5 to 55.6) in the per-protocol population. Injection-site pain was significantly more frequent among subjects receiving quadrivalent HPV vaccine than among those receiving placebo (57\% vs. $51 \%, \mathrm{P}<0.001)$.

CONCLUSIONS-Quadrivalent HPV vaccine prevents infection with HPV-6, 11, 16, and 18 and the development of related external genital lesions in males 16 to 26 years of age. (Funded by Merck and others; ClinicalTrials.gov number, NCT00090285.)

Human papillomavirus (HPV) infects the squamous epithelium in both sexes, leading to anogenital condylomata acuminata and, in males, to cancers of the penis, anus, and oropharynx. ${ }^{1}$ The rate of genital HPV infection among males is similar to that in females. In any 12 -month period, the probability that a sexually active male will acquire a new genital HPV infection is 0.29 to 0.39 per 1000 person-months,${ }^{2-4}$ which is similar to estimates for females. However, there are differences between the sexes in the immune response to HPV. A larger proportion of females are HPV-seropositive (17.9\%, vs. $7.9 \%$ of males), and females have higher titers of antibodies. ${ }^{5,6}$ The lower immune response to natural infection in males may partially explain the higher prevalence of HPV infections ${ }^{2,5,7}$ as compared with the prevalence among females, and the constant prevalence and incidence of HPV infection across a wide age range in males. ${ }^{2,4}$

The quadrivalent HPV vaccine, which is active against HPV types 6,11,16, and 18, is efficacious in preventing persistent infection and genital disease caused by these HPV types in females.${ }^{8-11}$ Similarly, HPV vaccination in males has the potential to significantly reduce HPV-associated anogenital infection and disease. Here we describe the results of a study of the efficacy of the quadrivalent HPV vaccine against anogenital infection and external genital lesions associated with HPV-6, 11, 16, or 18 in boys and men between the ages of 16 and 26 years.

\section{METHODS}

SUBJECTS

Between September 3, 2004, and August 29, 2008, we enrolled 4065 healthy boys and men from 71 sites in 18 countries in a randomized, placebo-controlled, double-blind study. A total of 3463 of the subjects were heterosexual (i.e., they reported that their sexual partners were exclusively female), and 602 had sex with male partners (i.e., they reported that they had engaged in insertive or receptive anal intercourse or oral sex with a male partner within the previous year). The median follow-up period, after administration of the first dose of vaccine or placebo, was 2.9 years.

For the heterosexual subjects, eligibility criteria were an age between 16 and 23 years and one to five female sexual partners during their lifetime; for the subjects who had sex with male partners, the criteria were an age between 16 and 26 years and one to five male or female partners during their lifetime. Subjects who had clinically detectable anogenital warts or genital lesions at screening that were suggestive of infection with non-HPV sexually transmitted diseases, or who had a history of such findings, were excluded.

\section{STUDY DESIGN AND OVERSIGHT}

Subjects were randomly assigned in a 1:1 ratio to receive quadrivalent HPV vaccine or placebo at day 1, month 2 ( \pm 3 weeks), and month 6 ( \pm 4 weeks). Vaccine or placebo was 
administered as a $0.5-\mathrm{ml}$ injection in the deltoid muscle (with all three doses administered in the same arm). The quadrivalent HPV L1 vaccine (Gardasil or Silgard, Merck) contains HPV-6, 11, 16, and 18 viruslike particles conjugated to an amorphous aluminum hydroxyphosphate sulfate (AAHS) adjuvant; the vaccine and the visually indistinguishable AAHS-containing placebo have been described previously. ${ }^{12}$ Details on randomization and blinding are provided in the Supplementary Appendix, available with the full text of this article at NEJM.org.

The trial was designed by the study sponsor, Merck, in collaboration with external investigators and an external data and safety monitoring board. The sponsor collated the data, monitored the conduct of the trial, performed statistical analyses, and coordinated the writing of the manuscript with all the authors. The academic authors were actively involved in the collection, analysis, and interpretation of the data; the initial drafting and revision of the manuscript for intellectual content; and the approval of the final manuscript. The first draft was written by the first author, with contributions from other authors. All authors vouch for the completeness and accuracy of the data presented. All authors had access to all the study data (with confidentiality agreements) and participated in the decision to submit the manuscript for publication.

The trial was conducted in accordance with the protocol (available at NEJM.org), which was approved by the institutional review boards at participating centers. Written informed consent was obtained from all subjects. At each study site, the trial was conducted in conformity with applicable country or local requirements regarding ethics committee review, informed consent, and other statutes or regulations regarding the protection of the rights and welfare of human subjects participating in biomedical research.

\section{STUDY POPULATIONS FOR ANALYSES}

Analyses were conducted in an intention-to-treat population consisting of subjects who received one or more doses of vaccine or placebo and returned for follow-up. These subjects, who might have been seropositive at enrollment or might have had positive results for the quadrivalent HPV vaccine types on polymerase-chain-reaction (PCR) assay, represented the general population of unvaccinated boys and men. A total of 175 subjects did not return for follow-up after receiving one dose of vaccine or placebo ( 82 in the vaccine group and 93 in the placebo group). Case counting in the intention-to-treat population commenced after day 1.

An efficacy analysis was also conducted in the per-protocol population - that is, subjects who were seronegative on day 1 and PCR-negative for both swab and biopsy specimens from day 1 through month 7 for the relevant vaccine HPV type (or types) and did not have any protocol violations (see Table S1 in the Supplementary Appendix). Subjects in the perprotocol population received all 3 vaccinations within 1 year and had 1 or more follow-up visits after month 7. Case counting commenced at month 7.

Additional analyses were conducted in a population of subjects who were negative for HPV-6, 11, 16, 18, 31, 33, 35, 39, 45, 51, 52, 56, 58, and 59 DNA and were seronegative for HPV-6, 11, 16, and 18 at enrollment and who received at least one dose of vaccine or placebo. This population approximates a population of young men before sexual debut. Case counting in this population began after day 1 .

\section{STUDY OBJECTIVES AND MEASURES OF EFFICACY}

The primary efficacy objective was to show that the quadrivalent HPV vaccine reduced the incidence of external genital lesions associated with HPV-6, 11, 16, or 18, as compared with placebo. The secondary efficacy objectives were to show that the vaccine reduced the 
incidence of persistent infection with these HPV types and the detection at any time of DNA associated with these viral types, as compared with placebo. We also analyzed the composite efficacy of the vaccine against the development of external genital lesions related to any HPV type (including HPV types that were identified with the use of a PCR assay [HPV-6, $11,16,18,31,33,35,39,45,51,52,56,58$, and 59] and types that were not so identified).

Detailed anogenital examinations were performed on day 1 and at months 7, 12, 18, 24, 30, and 36. Biopsies were performed for external genital lesions judged by the investigator to be possibly, probably, or definitely related to HPV and for any lesion whose cause was not known. Repeat biopsy of recurrent lesions (occurring within 2 months after the previous lesion, at the same location and with the same appearance) was not performed in order to avoid overestimation of incident external genital lesions. All biopsy specimens were processed independently to prevent contamination of HPV DNA and were assessed in a blinded fashion, first for the purpose of clinical management by pathologists at the central laboratory (Diagnostic Cytology Laboratories) and then for end-point adjudication by a fourmember panel of pathologists. ${ }^{8,9}$ Panel members disagreed on the interpretation of $0.3 \%$ of the biopsy specimens and resolved the problem by meeting to obtain a consensus. Clinical management was performed according to local standards of care. HPV testing of thin sections was performed at a central laboratory with the use of multiplex PCR assay. 8,9

Specimens for HPV testing were collected separately from the penis, scrotum, and perineal and perianal regions with the use of a nail file and Dacron swab on day 1 and at months 7 , $12,18,24,30$, and 36 . In the group of subjects who had sex with male partners, intra-anal specimens were collected with a Dacron swab. All specimens were tested for HPV DNA to identify subjects who were infected before enrollment and those who acquired new HPV infections during the study. Each thin-section and swab specimen was evaluated with three different primer-pair sets per HPV type, which amplified a portion of three separate open reading frames. ${ }^{13}$ Less than $5 \%$ of the PCR-positive biopsy specimens from external genital lesions ( 11 of 268 positive specimens) were positive for only 1 of 3 genes, and less than $4 \%$ of all specimens from external genital lesions (151 of 4886) could not be amplified to at least 1 of 14 HPV types tested.

To allow for assessment of vaccine safety, subjects recorded (on vaccination report cards) oral temperature and any adverse events occurring at the injection site on days 1 through 5 after receiving each dose of vaccine or placebo. They also recorded systemic adverse events and all serious adverse events that occurred on days 1 through 15 after receiving each dose. All serious adverse events that investigators believed to be associated with the vaccine or the study procedure and all deaths were recorded during the entire study period.

\section{END POINTS}

The primary end point of the study was the presence or absence of external genital lesions associated with HPV-6, 11, 16, or 18, defined as condylomata acuminata (external genital warts); penile, perianal, or perineal intraepithelial neoplasia (PIN); or penile, perianal, or perineal cancer. All other external genital lesions were also recorded, whether or not they contained an HPV type identified on PCR assay (HPV-6, 11, 16, 18, 31, 33, 35, 39, 45, 51, $52,56,58$, or 59). Primary end-point cases were confirmed by means of a consensual diagnosis of condyloma acuminatum, PIN grade 1 or grade $2-3$, or penile, perianal, or perineal cancer by the pathology panel after examination of a biopsy specimen and by detection of HPV DNA in an adjacent section of the same tissue block with the use of a PCR assay.

Persistent infection was defined as detection of the same HPV type $(6,11,16$, or 18$)$ in an anogenital swab or biopsy specimen collected on two or more consecutive visits, with an 
interval of at least 6 months ( \pm 1 month) between the visits. DNA detection was defined as detection of HPV 6,11,16, or 18 DNA in any clinical specimen on one or more visits (with visits made at any time during the study).

\section{STATISTICAL ANALYSIS}

To evaluate the primary end point, a fixed-event design was used. Assuming that the true efficacy of the vaccine is $80 \%, 32$ cases would provide at least $90 \%$ power to demonstrate an efficacy of more than $20 \%$ at a one-sided alpha level of 0.025 , and 23 cases in the heterosexual group would provide more than $90 \%$ power to demonstrate that vaccine efficacy is more than $0 \%$ in that subgroup. Therefore, the primary analysis was conducted when at least 32 cases that satisfied the primary end point had been observed in the entire study population and when at least 23 cases had been diagnosed among the heterosexual subgroup. The statistical criterion for study success required the lower bound of the confidence interval for vaccine efficacy to exclude $20 \%$. Vaccine efficacy was defined as $100 \% \times\left(1-\left[r_{v} / r_{p}\right]\right)$, with $r_{v}$, the incidence rate among vaccine recipients, defined as $C_{v}$, the number of primary efficacy cases among vaccine recipients, divided by $\tau_{\mathrm{v}}$, the total personyears of follow-up among vaccine recipients. Similarly, $r_{p}$, the incidence rate among placebo recipients, was defined as $\mathrm{C}_{\mathrm{p}}$, the number of primary efficacy cases among placebo recipients, divided by $\tau_{\mathrm{p}}$, the total person-years of follow-up among placebo recipients. The hypotheses related to vaccine efficacy were tested by constructing a two-sided exact confidence interval for vaccine efficacy under the assumption that the number of end-point cases among vaccine recipients followed a binomial distribution.

The confidence interval reported for the end point of persistent infection with HPV-6, 11, 16 , or 18 in the per-protocol population (97.5\%) differs from that reported in other analyses (95\%) because of application of the Hochberg multiplicity adjustment. This adjustment was necessary because of the two secondary end points: persistent infection and DNA detection. The adjustment was made so that the combined alpha level of the hypothesis tests for these two end points would not exceed a one-sided alpha level of 0.025 .

\section{RESULTS}

\section{CHARACTERISTICS OF THE SUBJECTS}

A total of 4164 boys and men were screened for the study, and 4065 were enrolled; 2032 were randomly assigned to the vaccine group and 2033 to the placebo group. (The reasons for exclusion from the study are presented in Table S1 in the Supplementary Appendix.) The study groups were balanced with respect to age, race or ethnic group, region, smoking status, circumcision status, and sexual history (Table S2 in the Supplementary Appendix).

A total of 4055 subjects received one or more doses of vaccine or placebo. Of these subjects, 2805 were eligible for the per-protocol population, with 1397 receiving the quadrivalent HPV vaccine and 1408 receiving placebo. The vaccine and placebo groups were similar with regard to reasons for discontinuing study participation and eligibility for inclusion in per-protocol analyses (Fig. S1 in the Supplementary Appendix).

\section{EFFICACY}

Within 1 month after administration of the third dose of vaccine, seroconversion for HPV-6, 11,16 , and 18 occurred in at least $97.4 \%$ of vaccinated subjects. The majority of subjects who were initially negative for all four vaccine HPV types (966 of 991 subjects [97.5\%]) had seroconversion for all four types by one month after the third dose. Nine subjects $(0.9 \%)$ did not undergo seroconversion to any of the four HPV types, and 15 subjects (1.5\%) underwent seroconversion to three of the four HPV types. Levels of anti-HPV antibodies 
peaked at month 7, with 447 milli-Merck units (mMU) per milliliter for HPV-6, $624 \mathrm{mMU}$ per milliliter for HPV-11, $2403 \mathrm{mMU}$ per milliliter for HPV-16, and $402 \mathrm{mMU}$ per milliliter for HPV-18 (milli-Merck units are defined in the Supplementary Appendix).

In the intention-to-treat population, 36 external genital lesions were seen in the vaccine group, as compared with 89 in the placebo group, resulting in an observed efficacy of $60.2 \%$ (95\% confidence interval [CI], 40.8 to 73.8) (Table 1 and Fig. 1). Vaccine efficacy against lesions related to HPV-6, 11, 16, or 18 was $65.5 \%$ (95\% CI, 45.8 to 78.6) (Table 1 and Fig. 1). There were significant reductions in the number of external genital lesions associated with HPV-6 (59.4\%; 95\% CI, 31.2 to 76.8) and HPV-11 (76.3\%; 95\% CI, 40.8 to 92.0). The vaccine was associated with nonsignificant reductions in external genital lesions associated with HPV-16 (70.3\%; 95\% CI, -5.5 to 94.7) and HPV-18 (33.9\%; 95\% CI, -476.7 to 94.5). Efficacy against condylomata acuminata associated with HPV-6 and HPV-11 was $67.2 \%$ (95\% CI, 47.3 to 80.3). Vaccine efficacy against PIN lesions was not observed in the intention-to-treat population. All PIN lesions were treated in accordance with the local standard of care.

In the per-protocol population, 6 external genital lesions were observed in the vaccine group and 36 in the placebo group, resulting in an observed efficacy of $83.8 \%$ (95\% CI, 61.2 to 94.4) (Table 2). Efficacy against external genital lesions associated with HPV types 6, 11, 16, or 18 was $90.4 \%$ (95\% CI, 69.2 to 98.1) (Table 2 and Fig. 1). Efficacy did not vary according to baseline characteristics. Efficacy against external genital lesions was $92.4 \%$ among heterosexual subjects and $79.0 \%$ among subjects who had sex with male partners, reaching statistical significance only among heterosexual subjects. The majority of such lesions were related to HPV types 6 and 11, with an observed vaccine efficacy of $84.3 \%$ (95\% CI, 46.5 to 97.0 ) and $90.9 \%$ (95\% CI, 37.7 to 99.8), respectively. No lesions associated with HPV-16 or HPV-18 were observed in the vaccine group, whereas two lesions associated with HPV-16 and one associated with HPV-18 were observed in the placebo group. The majority of external genital lesions observed were condylomata acuminata, and the observed vaccine efficacy against this lesion type was $89.4 \%$ (95\% CI, 65.5 to 97.9). No cases of PIN grade 1 or PIN grades 2 or 3 were observed in the vaccine group, but three cases of PIN grade 1 or worse were observed in the placebo group. High vaccine efficacy against external genital lesions was also observed in the group that was negative for $14 \mathrm{HPV}-\mathrm{DNA}$ types and seronegative for all vaccine types $(75.5 \%$; $95 \% \mathrm{CI}$, 54.3 to 87.7) (Table S3A and Fig. S2 in the Supplementary Appendix).

In the intention-to-treat population, the quadrivalent HPV vaccine significantly reduced the collective incidence of persistent infection with the four HPV-vaccine types, with an observed efficacy of $47.8 \%$ (95\% CI, 36.0 to 57.6) (Table 3). The vaccine also reduced the incidence of persistent infection with each individual HPV-vaccine type, with efficacy ranging from $44.7 \%$ for HPV-6 (95\% CI, 24.1 to 60.1) to 59.4\% for HPV-11 (95\% CI, 25.7 to 78.8). The quadrivalent HPV vaccine also significantly reduced DNA detection of individual HPV types at any time in the intention-to-treat population, with an efficacy range of $28.0 \%$ (95\% CI, 12.9 to 40.7) for HPV 16 to $43.2 \%$ (95\% CI, 18.7 to 60.7) for HPV 11.

In the per-protocol population, an overall reduction of $85.6 \%$ (97.5\% CI, 73.4 to 92.9$)$ in persistent infection with HPV-6, 11, 16, or 18 was observed. The observed efficacy of the vaccine against persistent infection with specific HPV types ranged from 78.7\% for HPV-16 (95\% CI, 55.5 to 90.9 ) to $96.0 \%$ for HPV-18 (95\% CI, 75.6 to 99.9). The vaccine was also efficacious in reducing detection of DNA at any time for all four HPV types (efficacy, 44.7\%; 95\% CI, 31.5 to 55.6). Reductions in DNA detection of individual HPV types were significant, with a range in efficacy of $41.1 \%$ for HPV 16 (95\% CI, 18.5 to 57.7$)$ to $62.1 \%$ for HPV 18 (95\% CI, 39.2 to 77.1). (Vaccine efficacy against persistent HPV infection and 
detection of HPV DNA in the per-protocol population is shown in Table S4 in the Supplementary Appendix.) In the population of subjects who were initially seronegative and DNA-negative for all tested HPV types, efficacy against persistent infection with HPV-6, 11,16 , or 18 was $68.3 \%$ (95\% CI, 57.1 to 76.9), and efficacy against DNA detection was $34.2 \%$ (95\% CI, 22.7 to 44.2) (Table S3B in the Supplementary Appendix).

\section{ADVERSE EVENTS}

Table 4 shows the distribution of clinical adverse events reported during the study period. Approximately $69 \%$ of the subjects in the vaccine group and $64 \%$ of those in the placebo group reported one or more adverse events. The majority of these events were related to the injection and were more common in the vaccine group than in the placebo group. An increase in oral temperature to $37.8^{\circ} \mathrm{C}$ or more was reported for $6.0 \%$ of subjects receiving the vaccine and $5.8 \%$ of subjects receiving placebo on the day of vaccination or up to 5 days after vaccination $(\mathrm{P}=0.82)$. Significantly more subjects in the vaccine group than in the placebo group reported injection-site pain (Table S5 in the Supplementary Appendix). Few subjects $(1.3 \%$ in the vaccine group and $1.0 \%$ in the placebo group) reported that this pain was "severe." (Mild pain was defined as being aware of a sign or symptom that was easily tolerated, moderate pain as causing enough discomfort to interfere with daily activities, and severe pain as causing incapacitation.) Approximately $14 \%$ of both vaccine (14.1\%) and placebo (14.6\%) recipients reported vaccine-related systemic adverse events; no serious adverse events related to vaccination were reported. (See Table 4 for a list of serious adverse events and Table S5 in the Supplementary Appendix for details of systemic adverse events and events related to the injection site.)

\section{DISCUSSION}

This study shows that prophylactic administration of quadrivalent HPV vaccine is efficacious in preventing the development of external genital lesions associated with infection with HPV-6, 11, 16, or 18 in boys and men 16 to 26 years of age. In the intentionto-treat population, efficacy against lesions related to HPV-6, 11, 16, or 18 was $65.5 \%$ (95\% CI, 45.8 to 78.6), and efficacy in preventing the development of any external genital lesion, regardless of HPV type, was $60.2 \%$ (95\% CI, 40.8 to 73.8). In the per-protocol population, the vaccine reduced the incidence of external genital lesions related to HPV-6, 11, 16, or 18 by $90.4 \%$ (95\% CI, 69.2 to 98.1 ). Efficacy against condylomata acuminata in the perprotocol population was $89.4 \%$ (95\% CI, 65.5 to 97.9). In addition, the quadrivalent HPV vaccine was efficacious against persistent infection with HPV-6, 11, 16, or 18 and against detection of DNA for these HPV types. Three cases of vaccine-related external genital lesions were observed among vaccine recipients in the per-protocol population. These cases may represent true vaccine failures, false negative results of HPV DNA or antibodydetection tests at baseline, or failure to identify these lesions at baseline, resulting in misclassification of subjects who were not truly members of the per-protocol population.

The proportion of subjects who reported one or more serious adverse events or who discontinued vaccination because of an adverse event was similar in the two study groups. As compared with rates of adverse events in studies involving girls and women, ${ }^{8,9}$ the rates in this study of boys and men were lower, particularly for systemic events and events related to the injection site. The lower rates among boys and men may be due to greater muscle mass at the injection site, a reluctance to report events perceived as minor, or both.

Our findings point to the efficacy of the quadrivalent HPV vaccine in preventing HPV infection and related diseases in men. Condylomata acuminata, the most common HPVrelated lesion, is associated with substantial physical and psychological morbidity ${ }^{14}$ and has a high rate of treatment failure, and treatment of recurrent episodes is costly. ${ }^{15}$ The results of 
this trial suggest that prophylactic vaccination of boys and men with quadrivalent HPV vaccine may reduce the incidence of condylomata acuminata, as observed within 3 years after the introduction of a vaccination program in Australia. ${ }^{16}$ Although it is likely that the prevention of HPV infection will help prevent anogenital cancer, intraepithelial neoplasia, recurrent respiratory papillomatosis, and cancer of the oropharynx and HPV transmission, each of these potential outcomes must be directly demonstrated.

The strengths of the current study include the rigorous design of the trial and the inclusion of subjects from several countries, as well as both heterosexual subjects and subjects who had sex with male partners, resulting in a diverse study population. HPV detection was standardized, and sampling was conducted across a broad area of the genital tract, with tests for HPV DNA performed separately at each anatomical location. The limitations of the trial include the narrow age range of the subjects and the relatively short follow-up period. Subjects enrolled in this trial had no more than five lifetime sexual partners, which may have resulted in overrepresentation of subjects with a low likelihood of HPV exposure at baseline and a low likelihood of subsequent exposure, as compared with the general population. Although the point efficacy estimates for the boys and men in this study are numerically lower than those for girls and women in previous studies, the confidence intervals overlap, suggesting that vaccine efficacy may be similar for the two sexes.

\section{Supplementary Material}

Refer to Web version on PubMed Central for supplementary material.

\section{Acknowledgments}

Supported by Merck and by grants (M01-RR-00079 and UL1 RR024131, to Dr. Palefsky) from the National Center for Research Resources and by a grant (RO1 CA098803, to Dr. Giuliano) from the National Institutes of Health.

Drs. Giuliano, Ferris, Moreira, Penny, and Palefsky report receiving grant support from Merck, either personally or through their institution; Dr. Penny reports receiving grant support from GlaxoSmithKline; Dr. Goldstone reports receiving grant support from Qiagen; Drs. Giuliano, Ferris, Moreira, Hillman, and Chang report receiving speaking fees or fees for board membership from Merck; Dr. Moi reports that his institution has received funding from Merck; Dr. Penny reports having stock or stock options in AstraZeneca; Dr. Palefsky reports receiving consulting fees from GlaxoSmithKline; Drs. Giuliano, Palefsky, Goldstone, Moreira, Moi, and Chang report receiving travel reimbursement from Merck; Dr. Bryan reports having an approved, filed, or pending patent related to subject matter discussed in this article; and Dr. Bryan, Dr. Marshall, Dr. Vuocolo, Dr. Barr, Dr. Haupt, Mr. Radley, and Dr. Guris are employees of Merck and own Merck stock or stock options.

We thank all the study participants and all the investigators who enrolled subjects in the study.

\section{References}

1. International Agency for Research on Cancer. IARC monographs on the evaluation of carcinogenic risks to humans. Vol. 90. Lyon, France: IARC; 2007. Human papillomaviruses. http:// screening.iarc.fr/doc/mono90.pdf

2. Giuliano AR, Lu B, Nielson CM, et al. Age-specific prevalence, incidence, and duration of human papillomavirus infections in a cohort of 290 US men. J Infect Dis. 2008; 198:827-35. [PubMed: 18657037]

3. Partridge JM, Hughes JP, Feng Q, et al. Genital human papillomavirus infection in men: incidence and risk factors in a cohort of university students. J Infect Dis. 2007; 196:1128-36. [PubMed: 17955430]

4. Giuliano AR, Papenfuss MR, Fulp W, et al. Incidence and clearance of type specific human papillomavirus infections in men ages 18-70 years. Lancet. in press. 
5. Dunne EF, Nielson CM, Stone KM, Markowitz LE, Giuliano AR. Prevalence of HPV infection among men: a systematic review of the literature. J Infect Dis. 2006; 194:1044-57. [PubMed: 16991079]

6. Stone KM, Karem KL, Sternberg MR, et al. Seroprevalence of human papillomavirus type 16 infection in the United States. J Infect Dis. 2002; 186:1396-402. [PubMed: 12404154]

7. Giuliano AR, Lazcano-Ponce E, Villa LL, et al. The human papillomavirus infection in men study: human papillomavirus prevalence and type distribution among men residing in Brazil, Mexico, and the United States. Cancer Epidemiol Biomarkers Prev. 2008; 17:2036-43. [PubMed: 18708396]

8. Garland SM, Hernandez-Avila M, Wheeler CM, et al. Quadrivalent vaccine against human papillomavirus to prevent anogenital diseases. N Engl J Med. 2007; 356:1928-43. [PubMed: 17494926]

9. The FUTURE II Study Group. Quadrivalent vaccine against human papillomavirus to prevent highgrade cervical lesions. N Engl J Med. 2007; 356:1915-27. [PubMed: 17494925]

10. Wheeler CM, Kjaer SK, Sigurdsson K, et al. The impact of quadrivalent human papillomavirus (HPV; types 6,11,16, and 18) L1 virus-like particle vaccine on infection and disease due to oncogenic nonvaccine HPV types in sexually active women aged 16-26 years. J Infect Dis. 2009; 199:936-44. [PubMed: 19236277]

11. Brown DR, Kjaer SK, Sigurdsson K, et al. The impact of quadrivalent human papillomavirus (HPV; types 6, 11, 16, and 18) L1 virus-like particle vaccine on infection and disease due to oncogenic non-vaccine HPV types in generally HPV-naive women aged 16-26 years. J Infect Dis. 2009; 199:926-35. [PubMed: 19236279]

12. Villa LL, Costa RLR, Petta CA, et al. Prophylactic quadrivalent human papillomavirus (types 6, 11, 16 and 18) L1 virus-like particle vaccine in young women: a randomised double-blind placebo-controlled multicentre phase II efficacy trial. Lancet Oncol. 2005; 6:271-8. [PubMed: 15863374]

13. Bryan JT, Taddeo F, Skulsky D, et al. Detection of specific human papillomavirus types in paraffin-embedded sections of cervical carcinomas. J Med Virol. 2006; 78:117-24. [PubMed: 16299730]

14. Palefsky JM. HPV infection in men. Dis Markers. 2007; 23:261-72. [PubMed: 17627061]

15. Insinga RP, Dasbach EJ, Myers ER. The health and economic burden of genital warts in a set of private health plans in the United States. Clin Infect Dis. 2003; 36:1397-403. [PubMed: 12766834]

16. Fairley, C. Rapid decline in genital warts after the implementation of a national quadrivalent human papillomavirus vaccination program for young women. London: International Society for Sexually Transmitted Disease Research; 2009. 


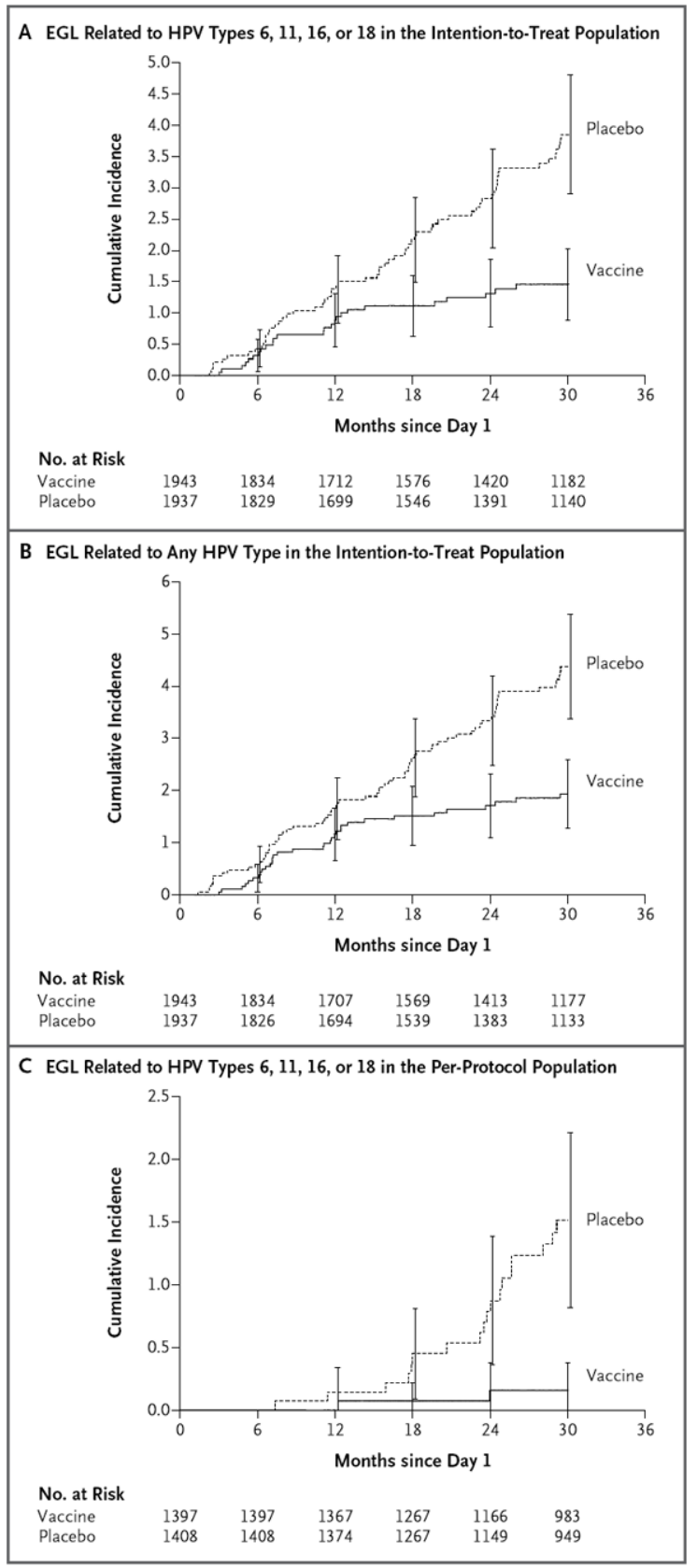

Figure 1. Analysis of the Time to Appearance of External Genital Lesions in the Intention-toTreat and Per-Protocol Populations

Panel A shows the incidence of external genital lesions (EGLs) associated with HPV types $6,11,16$, and 18 in the intention-to-treat population, Panel B the relation of EGLs to any HPV type in the intention-to-treat population, and Panel $\mathrm{C}$ the incidence of EGLs associated with HPV-6, 11, 16, and 18 in the per-protocol population. 


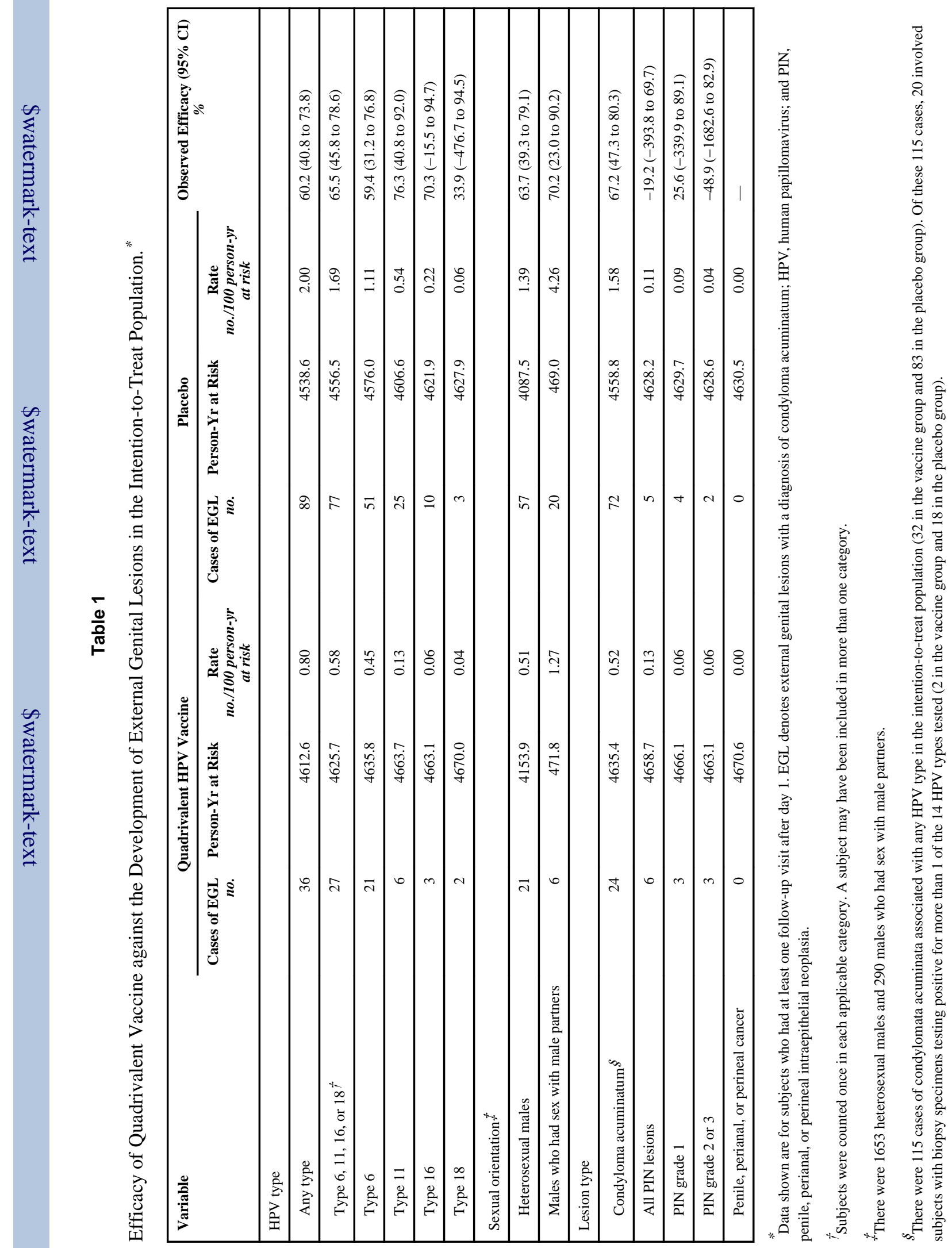




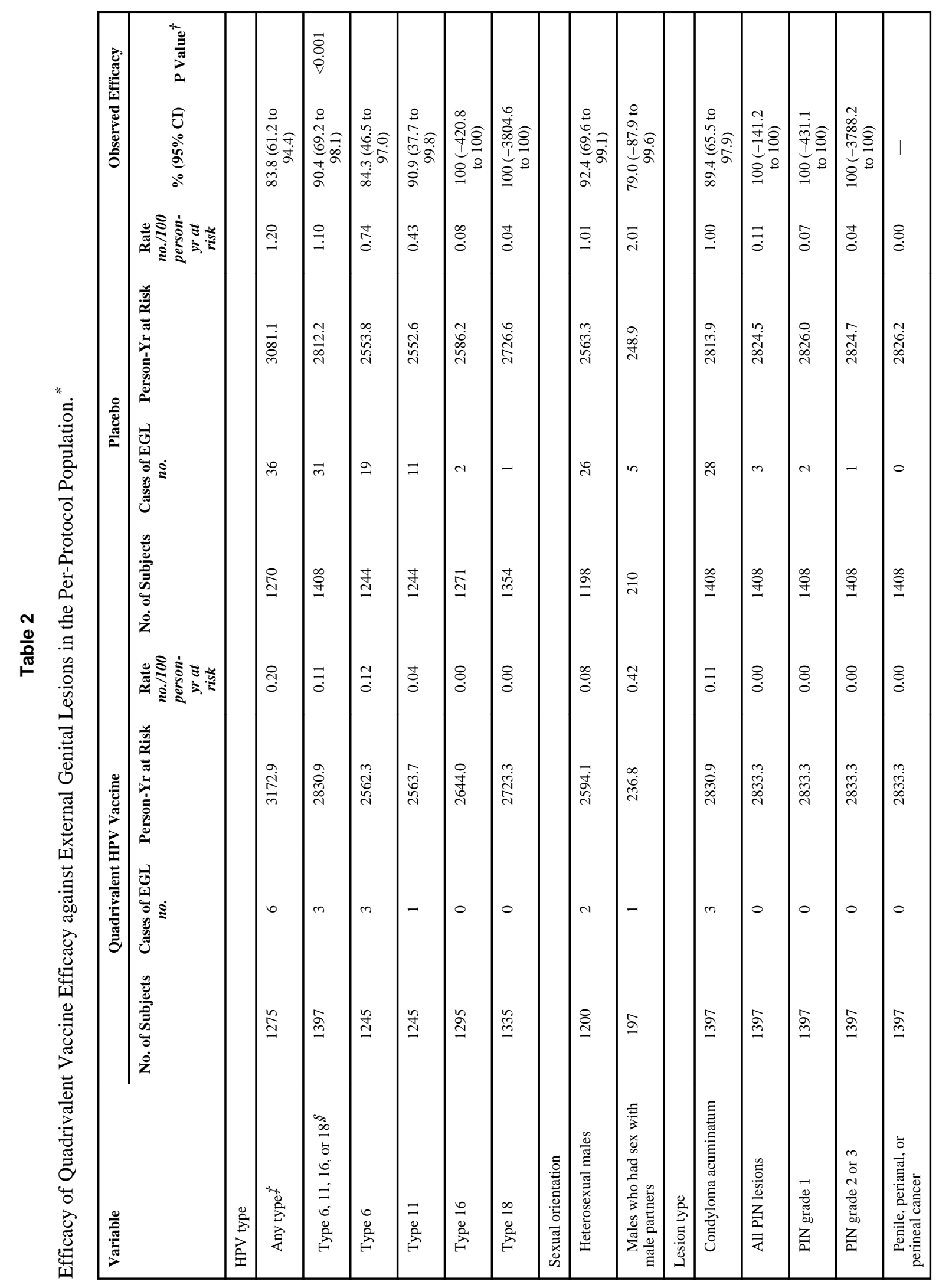




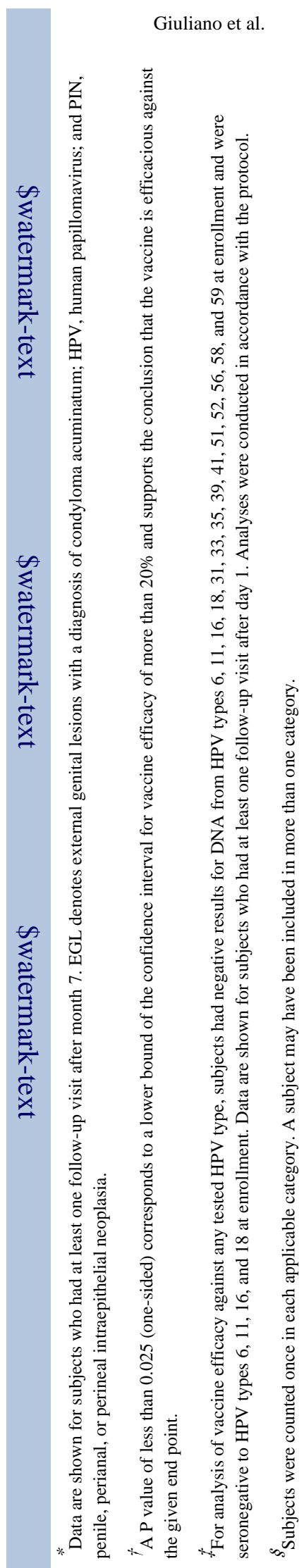

NEngl J Med. Author manuscript; available in PMC 2012 November 11. 


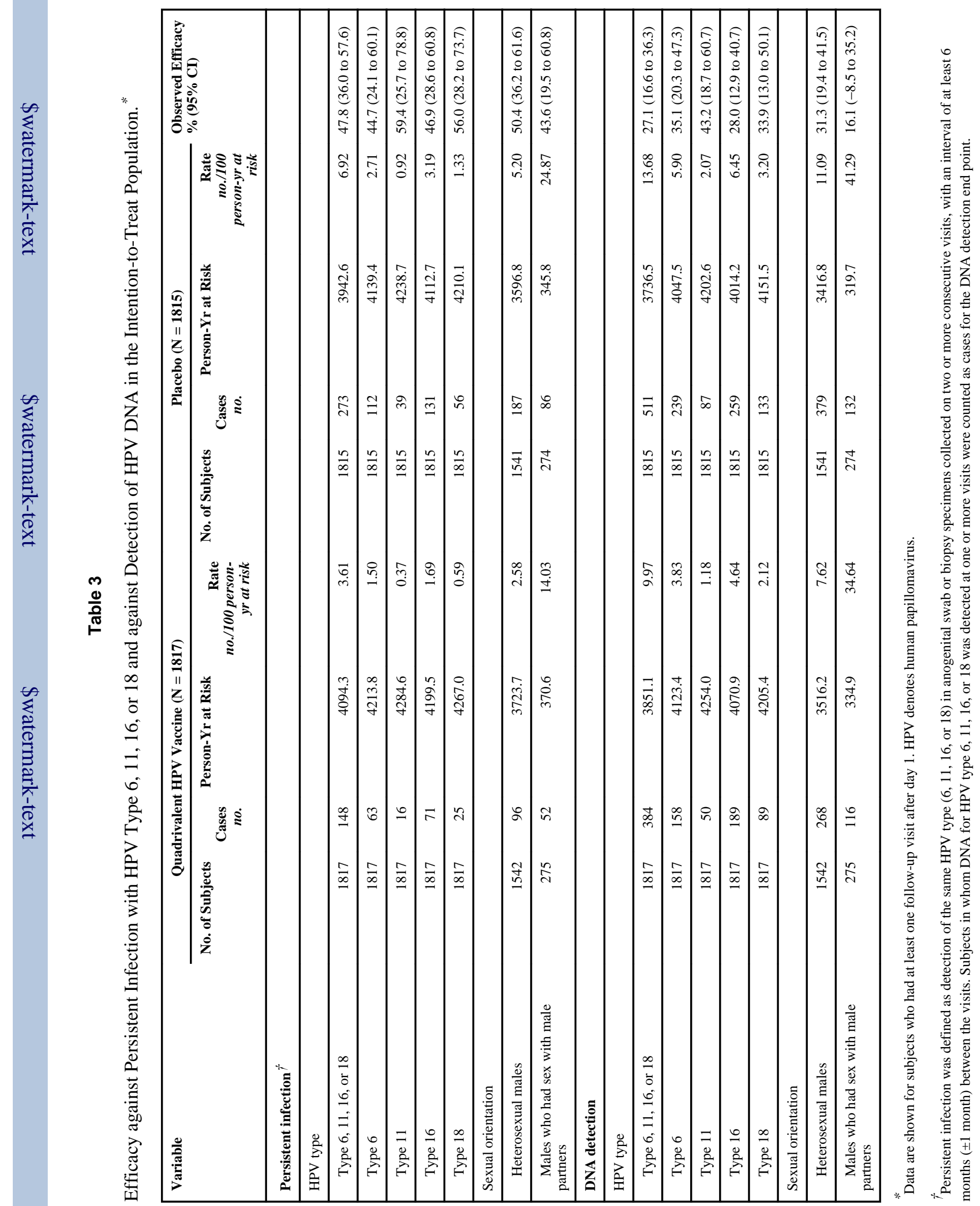

NEngl J Med. Author manuscript; available in PMC 2012 November 11. 


\section{Table 4}

Summary of Adverse Events.*

\begin{tabular}{|c|c|c|c|c|}
\hline \multirow[t]{2}{*}{ Adverse Event } & Quadrivalent HPV Vaccine & Placebo & \multirow{2}{*}{$\begin{array}{c}\text { Difference in Risk }(95 \% \text { CI) } \\
\text { percentage points }\end{array}$} & \multirow[t]{2}{*}{ P Value ${ }^{\dagger}$} \\
\hline & \multicolumn{2}{|l|}{ no. $(\%)$} & & \\
\hline No. of subjects & 2020 & 2029 & & \\
\hline No. of subjects with follow-up data & 1945 & 1950 & & \\
\hline \multicolumn{5}{|c|}{$\begin{array}{l}\text { Subjects with events during entire study } \\
\text { period }\end{array}$} \\
\hline No event & $599(30.8)$ & $698(35.8)$ & & \\
\hline One or more events & $1346(69.2)$ & $1252(64.2)$ & $5.0(2.0$ to 8.0$)$ & $<0.001$ \\
\hline Injection site & $1169(60.1)$ & $1047(53.7)$ & $6.4(3.3$ to 9.5$)$ & $<0.001$ \\
\hline Systemic & $616(31.7)$ & $622(31.9)$ & $-0.2(-3.2$ to 2.7$)$ & 0.88 \\
\hline Vaccine-related events ${ }^{t}$ & $1242(63.9)$ & $1134(58.2)$ & 5.7 (2.6 to 8.8$)$ & $<0.001$ \\
\hline Injection site & $1169(60.1)$ & $1046(53.6)$ & 6.5 (3.3 to 9.6$)$ & $<0.001$ \\
\hline Systemic & $274(14.1)$ & $284(14.6)$ & $-0.5(-2.7$ to 1.7$)$ & 0.67 \\
\hline Serious events $\mathcal{\xi}$ & $8(0.4)$ & $11(0.6)$ & $-0.2(-0.7$ to 0.3$)$ & 0.49 \\
\hline Serious vaccine-related events $t \xi$ & 0 & 0 & $0.0(-0.2$ to 0.2$)$ & 1.00 \\
\hline Death & $3(0.2)$ & $10(0.5)$ & $-0.4(-0.8$ to 0.01$)$ & 0.052 \\
\hline \multicolumn{5}{|c|}{$\begin{array}{l}\text { Subjects with adverse events in first } 15 \text { days } \\
\text { after injection }\end{array}$} \\
\hline No event & $600(30.8)$ & $706(36.2)$ & & \\
\hline One or more events & $1345(69.2)$ & $1244(63.8)$ & $5.4(2.4$ to 8.3$)$ & $<0.001$ \\
\hline Injection site & $1169(60.1)$ & $1047(53.7)$ & $6.4(3.3$ to 9.5$)$ & $<0.001$ \\
\hline Systemic & $615(31.6)$ & $613(31.4)$ & $0.2(-2.7$ to 3.1$)$ & 0.90 \\
\hline Vaccine-related events ${ }^{t}$ & $1242(63.9)$ & $1134(58.2)$ & 5.7 (2.6 to 8.8$)$ & $<0.001$ \\
\hline Injection site & $1169(60.1)$ & $1046(53.6)$ & 6.5 (3.3 to 9.6$)$ & $<0.001$ \\
\hline Systemic & $274(14.1)$ & $284(14.6)$ & $-0.5(-2.7$ to 1.7$)$ & 0.67 \\
\hline Serious events $\mathcal{\xi}$ & $5(0.3)$ & $1(0.1)$ & $0.2(-0.1$ to 0.6$)$ & 0.10 \\
\hline Serious vaccine-related events $t \xi$ & 0 & 0 & $0(-0.2$ to 0.2$)$ & 1.00 \\
\hline Death & 0 & 0 & $0(-0.2$ to 0.2$)$ & 1.00 \\
\hline
\end{tabular}

Percentages were calculated on the basis of the number of subjects with follow-up data. HPV denotes human papillomavirus.

${ }^{\dagger} \mathrm{P}$ values have not been adjusted for multiple comparisons.

Vaccine-related events were those determined by the investigator to be possibly, probably, or definitely related to the vaccine.

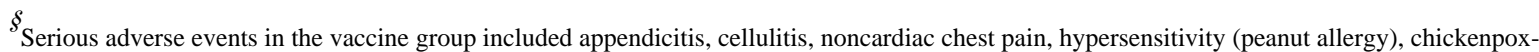
related seizure, traffic accident (there were two such accidents, both resulting in death), and gunshot wound (resulting in death). Serious adverse events in the placebo group included contusion related to traffic accident and the following fatal events: gunshot wound (in 3 subjects), drug overdose (2), suicide (2), traffic accident (1), chemical poisoning (1), and myocardial ischemia (1). Three additional subjects were considered to have serious adverse events because they received more than 3 doses of vaccine or placebo; none of these subjects had adverse events after any of the injections they received. 